Supplement of Hydrol. Earth Syst. Sci., 21, 4379-4401, 2017

https://doi.org/10.5194/hess-21-4379-2017-supplement

(C) Author(s) 2017. This work is distributed under

the Creative Commons Attribution 3.0 License.

(c) (1)

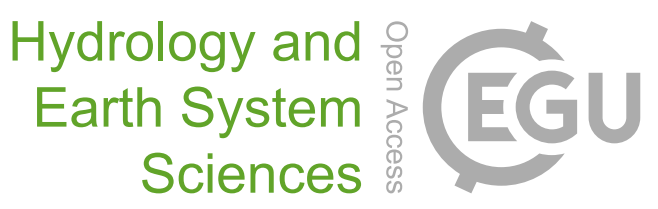

Supplement of

\title{
The effect of GCM biases on global runoff simulations of a land surface model
}

Lamprini V. Papadimitriou et al.

Correspondence to: Ioannis K. Tsanis (tsanis@hydromech.gr)

The copyright of individual parts of the supplement might differ from the CC BY 3.0 License. 
Table S 1. Basin station information

\begin{tabular}{llll}
\hline Basins & GRDC station number & GRDC station name & Area $\left[\mathrm{km}^{2}\right]$ \\
\hline Amazon & 3629001 & Obidos & 4680000 \\
\hline Congo & 1147010 & Kinshasa & 3475000 \\
\hline Mississippi & 4127800 & Vicksburg MS & 2964255 \\
\hline Lena & 2903430 & Stolb & 2460000 \\
\hline Volga & 6977100 & Volgograd power plant & 1360000 \\
\hline Ganges & 2646200 & Hardinge bridge & 846300 \\
\hline Danube & 6742900 & Ceatal Izmail & 807000 \\
\hline Elbe & 6340110 & Neu-Darchau & 131950 \\
\hline Kemijoki & 6854700 & Isohaara & 50686 \\
\hline
\end{tabular}


Table S 2. Values of input variables, for each GCM (GFDL, IPSL and MIROC), the ensemble mean (Ens.Mean) and WFDEI data, spatially averaged for 24 Giorgi regions.

\begin{tabular}{|c|c|c|c|c|c|c|c|c|c|c|}
\hline & \multicolumn{5}{|c|}{$\mathrm{P}[\mathrm{mm} / \mathrm{day}]$} & \multicolumn{5}{|l|}{$\mathrm{T}[\mathrm{K}]$} \\
\hline & GFDL & IPSL & MIROC & Ens.Mean & WFDEI & GFDL & IPSL & MIROC & Ens.Mean & WFDEI \\
\hline NEU & 2.61 & 2.30 & 2.53 & 2.48 & 2.43 & 277.90 & 277.15 & 281.16 & 278.74 & 279.50 \\
\hline MED & 1.44 & 1.08 & 1.44 & 1.32 & 1.56 & 288.73 & 287.57 & 290.13 & 288.81 & 288.26 \\
\hline NEE & 1.71 & 1.67 & 1.79 & 1.72 & 1.67 & 274.42 & 274.15 & 277.89 & 275.49 & 276.75 \\
\hline NAS & 1.59 & 1.66 & 1.78 & 1.68 & 1.25 & 267.91 & 269.70 & 270.71 & 269.44 & 267.53 \\
\hline CAS & 0.92 & 0.79 & 1.36 & 1.02 & 0.93 & 284.84 & 284.00 & 287.60 & 285.48 & 285.79 \\
\hline TIB & 1.36 & 1.05 & 1.99 & 1.47 & 0.63 & 274.44 & 271.79 & 273.20 & 273.14 & 275.70 \\
\hline EAS & 2.96 & 2.88 & 2.96 & 2.94 & 2.57 & 286.26 & 285.73 & 288.39 & 286.79 & 284.48 \\
\hline SEA & 8.77 & 6.74 & 6.80 & 7.44 & 6.96 & 299.45 & 299.15 & 298.99 & 299.19 & 299.21 \\
\hline NAU & 2.97 & 1.37 & 3.46 & 2.60 & 1.65 & 297.80 & 297.47 & 298.36 & 297.87 & 297.40 \\
\hline SAU & 1.79 & 1.60 & 2.28 & 1.89 & 1.28 & 289.28 & 286.59 & 287.49 & 287.79 & 290.68 \\
\hline SAH & 0.22 & 0.06 & 0.35 & 0.21 & 0.15 & 297.02 & 294.15 & 296.73 & 295.97 & 298.18 \\
\hline WAF & 4.60 & 2.92 & 4.02 & 3.85 & 2.86 & 298.65 & 298.50 & 299.70 & 298.95 & 300.57 \\
\hline EAF & 2.15 & 1.52 & 2.87 & 2.18 & 1.99 & 297.86 & 297.14 & 298.09 & 297.69 & 298.99 \\
\hline EQF & 2.87 & 3.34 & 2.80 & 3.00 & 2.67 & 295.21 & 295.47 & 295.61 & 295.43 & 296.00 \\
\hline SQF & 3.33 & 3.18 & 2.79 & 3.10 & 3.04 & 295.89 & 295.95 & 296.37 & 296.07 & 295.96 \\
\hline SAF & 2.37 & 1.62 & 2.20 & 2.06 & 1.27 & 291.60 & 290.33 & 290.83 & 290.92 & 290.89 \\
\hline WNA & 1.92 & 1.88 & 2.32 & 2.04 & 1.49 & 282.01 & 282.41 & 284.29 & 282.90 & 282.96 \\
\hline CNA & 2.48 & 2.11 & 2.12 & 2.23 & 2.62 & 283.22 & 283.91 & 286.66 & 284.59 & 284.58 \\
\hline ENA & 3.53 & 3.49 & 3.77 & 3.60 & 3.20 & 286.57 & 287.57 & 289.45 & 287.86 & 282.26 \\
\hline CAM & 3.43 & 2.17 & 2.22 & 2.60 & 2.84 & 295.70 & 295.89 & 297.40 & 296.33 & 295.32 \\
\hline AMZ & 3.57 & 3.55 & 4.06 & 3.72 & 5.32 & 297.74 & 297.44 & 297.66 & 297.61 & 297.94 \\
\hline CSA & 2.37 & 1.71 & 2.20 & 2.09 & 2.83 & 291.79 & 290.06 & 291.07 & 290.97 & 290.61 \\
\hline SSA & 2.58 & 2.76 & 2.70 & 2.68 & 2.57 & 281.71 & 278.10 & 279.75 & 279.85 & 281.32 \\
\hline \multirow[t]{3}{*}{ SAS } & 3.61 & 2.94 & 4.76 & 3.77 & 3.75 & 296.89 & 296.78 & 297.21 & 296.96 & 296.36 \\
\hline & \multicolumn{5}{|c|}{$\mathrm{R} 1[\mathrm{~W} / \mathrm{m} 2]$} & \multicolumn{5}{|c|}{ Rs [W/m2] } \\
\hline & GFDL & IPSL & MIROC & Ens.Mean & WFDEI & GFDL & IPSL & MIROC & Ens.Mean & WFDEI \\
\hline NEU & 298.76 & 289.91 & 313.39 & 300.69 & 295.33 & 106.90 & 113.95 & 105.91 & 108.92 & 115.03 \\
\hline MED & 325.96 & 306.36 & 328.73 & 320.35 & 314.19 & 194.08 & 207.62 & 202.13 & 201.27 & 199.11 \\
\hline NEE & 283.96 & 268.51 & 293.05 & 281.84 & 286.82 & 113.46 & 130.74 & 131.76 & 125.32 & 113.86 \\
\hline NAS & 255.12 & 250.35 & 261.36 & 255.61 & 245.13 & 115.27 & 125.07 & 132.40 & 124.24 & 117.66 \\
\hline CAS & 294.43 & 276.17 & 300.68 & 290.43 & 295.95 & 208.62 & 212.39 & 224.00 & 215.01 & 204.59 \\
\hline TIB & 254.00 & 226.63 & 239.74 & 240.12 & 239.85 & 193.41 & 203.32 & 238.30 & 211.68 & 216.40 \\
\hline EAS & 330.69 & 311.34 & 329.96 & 324.00 & 310.11 & 175.70 & 203.77 & 197.67 & 192.38 & 171.51 \\
\hline SEA & 412.92 & 398.55 & 404.30 & 405.26 & 415.89 & 217.69 & 235.62 & 220.55 & 224.62 & 194.56 \\
\hline NAU & 375.94 & 353.13 & 375.57 & 368.22 & 357.89 & 245.74 & 275.31 & 245.39 & 255.48 & 248.10 \\
\hline SAU & 330.27 & 314.19 & 326.86 & 323.77 & 326.54 & 197.93 & 190.86 & 185.11 & 191.30 & 216.98 \\
\hline SAH & 337.31 & 309.98 & 339.92 & 329.07 & 337.15 & 262.15 & 275.38 & 277.74 & 271.75 & 264.56 \\
\hline WAF & 384.32 & 363.56 & 388.70 & 378.86 & 392.92 & 230.64 & 281.46 & 240.12 & 250.74 & 231.51 \\
\hline
\end{tabular}




\begin{tabular}{|c|c|c|c|c|c|c|c|c|c|c|}
\hline EAF & 371.89 & 347.30 & 372.53 & 363.91 & 384.45 & 251.09 & 292.60 & 247.54 & 263.74 & 237.33 \\
\hline EQF & 372.31 & 356.07 & 365.27 & 364.55 & 377.08 & 240.21 & 278.16 & 231.80 & 250.05 & 232.56 \\
\hline SQF & 378.02 & 362.43 & 370.00 & 370.15 & 373.27 & 234.04 & 268.65 & 237.10 & 246.60 & 223.85 \\
\hline SAF & 344.64 & 323.67 & 334.37 & 334.23 & 321.71 & 217.70 & 237.28 & 219.01 & 224.66 & 232.14 \\
\hline WNA & 296.89 & 293.37 & 302.39 & 297.55 & 281.30 & 196.70 & 183.22 & 195.71 & 191.87 & 205.10 \\
\hline CNA & 311.69 & 298.60 & 310.79 & 307.03 & 308.70 & 178.09 & 198.56 & 207.13 & 194.59 & 185.28 \\
\hline ENA & 339.03 & 327.43 & 341.57 & 336.01 & 305.46 & 171.46 & 189.71 & 187.69 & 182.95 & 164.46 \\
\hline CAM & 377.27 & 360.63 & 370.16 & 369.35 & 366.67 & 229.89 & 252.57 & 248.63 & 243.70 & 229.00 \\
\hline AMZ & 386.81 & 370.84 & 385.43 & 381.03 & 410.20 & 236.57 & 276.72 & 229.83 & 247.71 & 195.18 \\
\hline CSA & 345.94 & 327.65 & 331.53 & 335.04 & 336.63 & 213.80 & 221.64 & 223.21 & 219.55 & 210.34 \\
\hline SSA & 306.49 & 300.96 & 309.79 & 305.75 & 296.61 & 143.79 & 119.23 & 129.33 & 130.78 & 149.19 \\
\hline \multirow[t]{3}{*}{ SAS } & 376.44 & 362.65 & 375.76 & 371.62 & 373.47 & 232.43 & 252.54 & 230.45 & 238.47 & 207.03 \\
\hline & \multicolumn{5}{|c|}{$\mathrm{H}[\mathrm{kg} / \mathrm{kg}]$} & \multicolumn{5}{|l|}{ Ps [HPa] } \\
\hline & GFDL & IPSL & MIROC & Ens.Mean & WFDEI & GFDL & IPSL & MIROC & Ens.Mean & WFDEI \\
\hline NEU & 0.0051 & 0.0048 & 0.0066 & 0.0055 & 0.0055 & 995.14 & 994.72 & 992.99 & 994.28 & 983.13 \\
\hline MED & 0.0075 & 0.0075 & 0.0087 & 0.0079 & 0.0076 & 981.06 & 979.10 & 980.40 & 980.19 & 958.26 \\
\hline NEE & 0.0042 & 0.0041 & 0.0054 & 0.0046 & 0.0045 & 998.58 & 997.13 & 995.35 & 997.02 & 994.48 \\
\hline NAS & 0.0031 & 0.0036 & 0.0042 & 0.0037 & 0.0033 & 966.94 & 964.29 & 964.13 & 965.12 & 955.25 \\
\hline CAS & 0.0044 & 0.0044 & 0.0057 & 0.0048 & 0.0055 & 900.50 & 896.25 & 899.36 & 898.70 & 893.06 \\
\hline TIB & 0.0033 & 0.0034 & 0.0042 & 0.0036 & 0.0034 & 735.65 & 728.50 & 736.90 & 733.68 & 734.45 \\
\hline EAS & 0.0090 & 0.0089 & 0.0108 & 0.0096 & 0.0078 & 974.67 & 969.55 & 973.25 & 972.49 & 947.43 \\
\hline SEA & 0.0176 & 0.0178 & 0.0186 & 0.0180 & 0.0176 & 1000.13 & 1001.34 & 1003.18 & 1001.55 & 977.85 \\
\hline NAU & 0.0121 & 0.0117 & 0.0140 & 0.0126 & 0.0096 & 991.65 & 994.78 & 994.03 & 993.49 & 978.92 \\
\hline SAU & 0.0079 & 0.0068 & 0.0081 & 0.0076 & 0.0071 & 1004.23 & 1001.10 & 1002.27 & 1002.53 & 988.15 \\
\hline SAH & 0.0061 & 0.0055 & 0.0068 & 0.0061 & 0.0061 & 965.67 & 965.58 & 966.70 & 965.98 & 955.18 \\
\hline WAF & 0.0132 & 0.0123 & 0.0145 & 0.0133 & 0.0124 & 982.76 & 982.58 & 982.96 & 982.77 & 970.86 \\
\hline EAF & 0.0113 & 0.0112 & 0.0130 & 0.0118 & 0.0122 & 939.81 & 936.28 & 940.58 & 938.89 & 928.97 \\
\hline EQF & 0.0126 & 0.0135 & 0.0132 & 0.0131 & 0.0131 & 927.28 & 923.68 & 927.22 & 926.06 & 897.12 \\
\hline SQF & 0.0134 & 0.0136 & 0.0144 & 0.0138 & 0.0123 & 964.04 & 963.95 & 964.50 & 964.16 & 924.14 \\
\hline SAF & 0.0104 & 0.0094 & 0.0104 & 0.0101 & 0.0077 & 970.87 & 970.37 & 970.88 & 970.71 & 909.10 \\
\hline WNA & 0.0059 & 0.0062 & 0.0074 & 0.0065 & 0.0051 & 908.11 & 909.20 & 907.96 & 908.42 & 867.44 \\
\hline CNA & 0.0071 & 0.0067 & 0.0078 & 0.0072 & 0.0071 & 970.30 & 967.75 & 964.45 & 967.50 & 967.64 \\
\hline ENA & 0.0092 & 0.0097 & 0.0113 & 0.0101 & 0.0068 & 1005.31 & 1003.65 & 1001.77 & 1003.58 & 986.35 \\
\hline CAM & 0.0135 & 0.0136 & 0.0147 & 0.0140 & 0.0122 & 983.62 & 983.88 & 982.98 & 983.49 & 928.03 \\
\hline AMZ & 0.0135 & 0.0140 & 0.0158 & 0.0144 & 0.0158 & 969.59 & 970.66 & 970.49 & 970.25 & 956.50 \\
\hline CSA & 0.0100 & 0.0091 & 0.0096 & 0.0096 & 0.0095 & 976.00 & 975.62 & 973.88 & 975.17 & 935.84 \\
\hline SSA & 0.0060 & 0.0047 & 0.0057 & 0.0055 & 0.0050 & 997.59 & 994.17 & 993.09 & 994.95 & 957.83 \\
\hline \multirow[t]{3}{*}{ SAS } & 0.0134 & 0.0136 & 0.0152 & 0.0141 & 0.0132 & 965.75 & 965.46 & 965.67 & 965.63 & 932.59 \\
\hline & \multicolumn{5}{|l|}{$\mathrm{W}[\mathrm{m} / \mathrm{s}]$} & & & & & \\
\hline & GFDL & IPSL & MIROC & Ens.Mean & WFDEI & & & & & \\
\hline NEU & 5.50 & 4.47 & 4.10 & 4.69 & 3.64 & & & & & \\
\hline MED & 4.02 & 3.99 & 4.32 & 4.11 & 3.17 & & & & & \\
\hline NEE & 3.61 & 2.93 & 3.01 & 3.18 & 3.56 & & & & & \\
\hline NAS & 3.57 & 3.46 & 3.85 & 3.63 & 3.05 & & & & & \\
\hline
\end{tabular}




\begin{tabular}{llllll|}
\hline CAS & 2.85 & 3.64 & 4.33 & 3.61 & 3.27 \\
\hline TIB & 2.46 & 3.98 & 5.50 & 3.98 & 3.49 \\
\hline EAS & 4.54 & 4.39 & 4.18 & 4.37 & 3.15 \\
\hline SEA & 5.09 & 3.75 & 3.89 & 4.24 & 1.83 \\
\hline NAU & 4.48 & 3.93 & 4.24 & 4.22 & 4.24 \\
\hline SAU & 6.46 & 6.87 & 7.14 & 6.83 & 4.16 \\
\hline SAH & 3.59 & 4.12 & 4.53 & 4.08 & 4.33 \\
\hline WAF & 2.84 & 2.54 & 3.12 & 2.83 & 2.77 \\
\hline EAF & 2.95 & 3.23 & 3.85 & 3.34 & 3.24 \\
\hline EQF & 3.08 & 2.75 & 3.19 & 3.01 & 2.68 \\
\hline SQF & 3.82 & 3.55 & 4.01 & 3.79 & 2.49 \\
\hline SAF & 5.15 & 5.40 & 5.78 & 5.44 & 3.79 \\
\hline WNA & 3.88 & 3.50 & 4.78 & 4.05 & 3.06 \\
\hline CNA & 3.29 & 3.28 & 3.34 & 3.30 & 3.90 \\
\hline ENA & 5.22 & 4.72 & 4.46 & 4.80 & 2.86 \\
\hline CAM & 4.48 & 3.89 & 4.55 & 4.31 & 2.50 \\
\hline AMZ & 2.91 & 2.73 & 2.10 & 2.58 & 1.71 \\
\hline CSA & 4.68 & 4.83 & 5.11 & 4.87 & 3.24 \\
\hline SSA & 7.94 & 7.90 & 8.54 & 8.12 & 5.14 \\
\hline SAS & 4.31 & 3.56 & 3.13 & 3.67 & 2.49 \\
\hline & & & & & \\
\hline
\end{tabular}



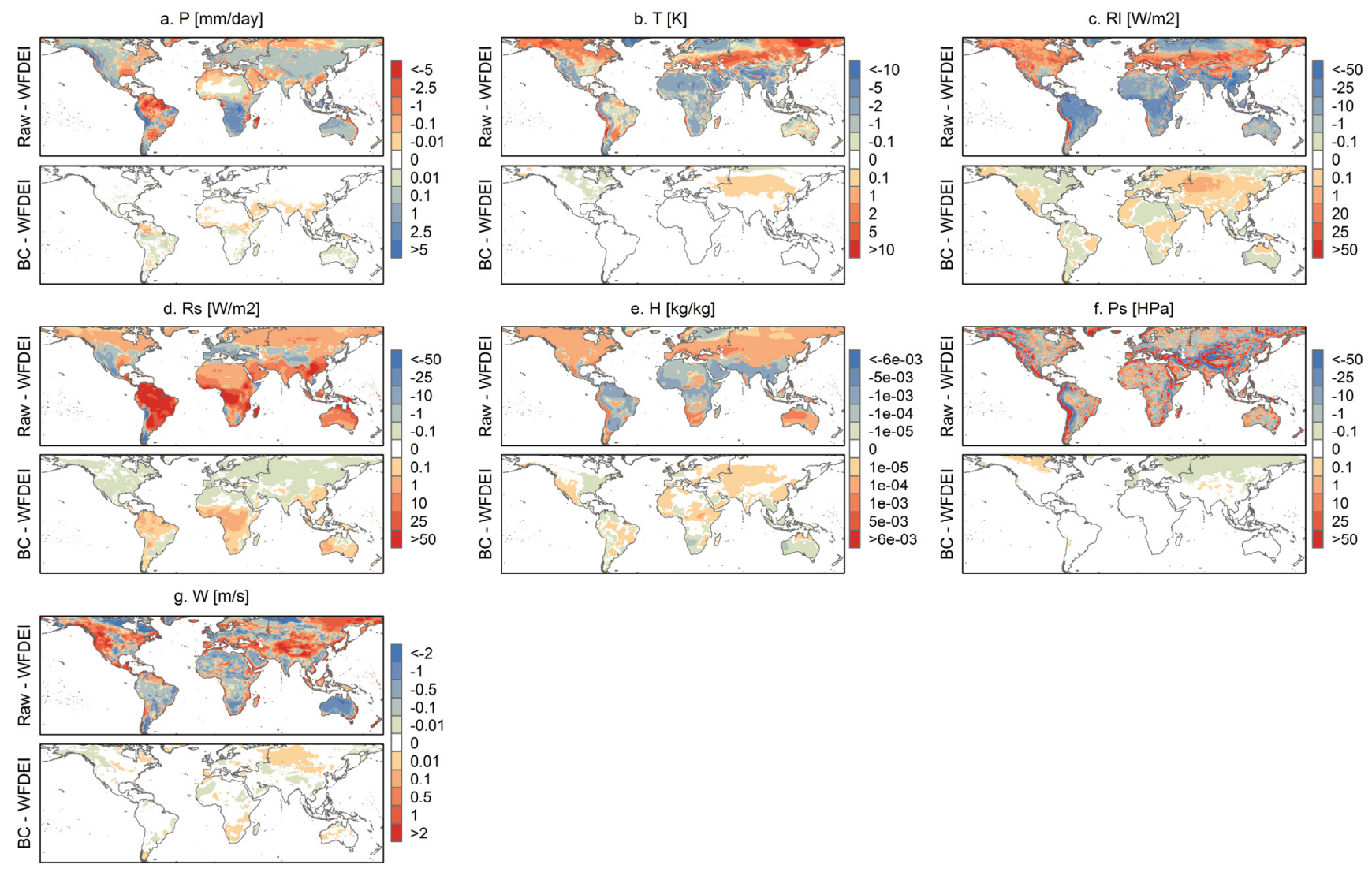

Figure S 1. Difference maps, showing initial (Raw-WFDEI) and remaining (BC-WFDEI) biases of the GCM ensemble forcing variables: a.Precipitation, b.Temperature, c.Longwave downward radiation, d.Shortwave downward radiation, e.Specific humidity, h.Surface pressure, g. Wind. Differences are calculated between the December-January-February averages (DJF) of the 1981-2010 period. 

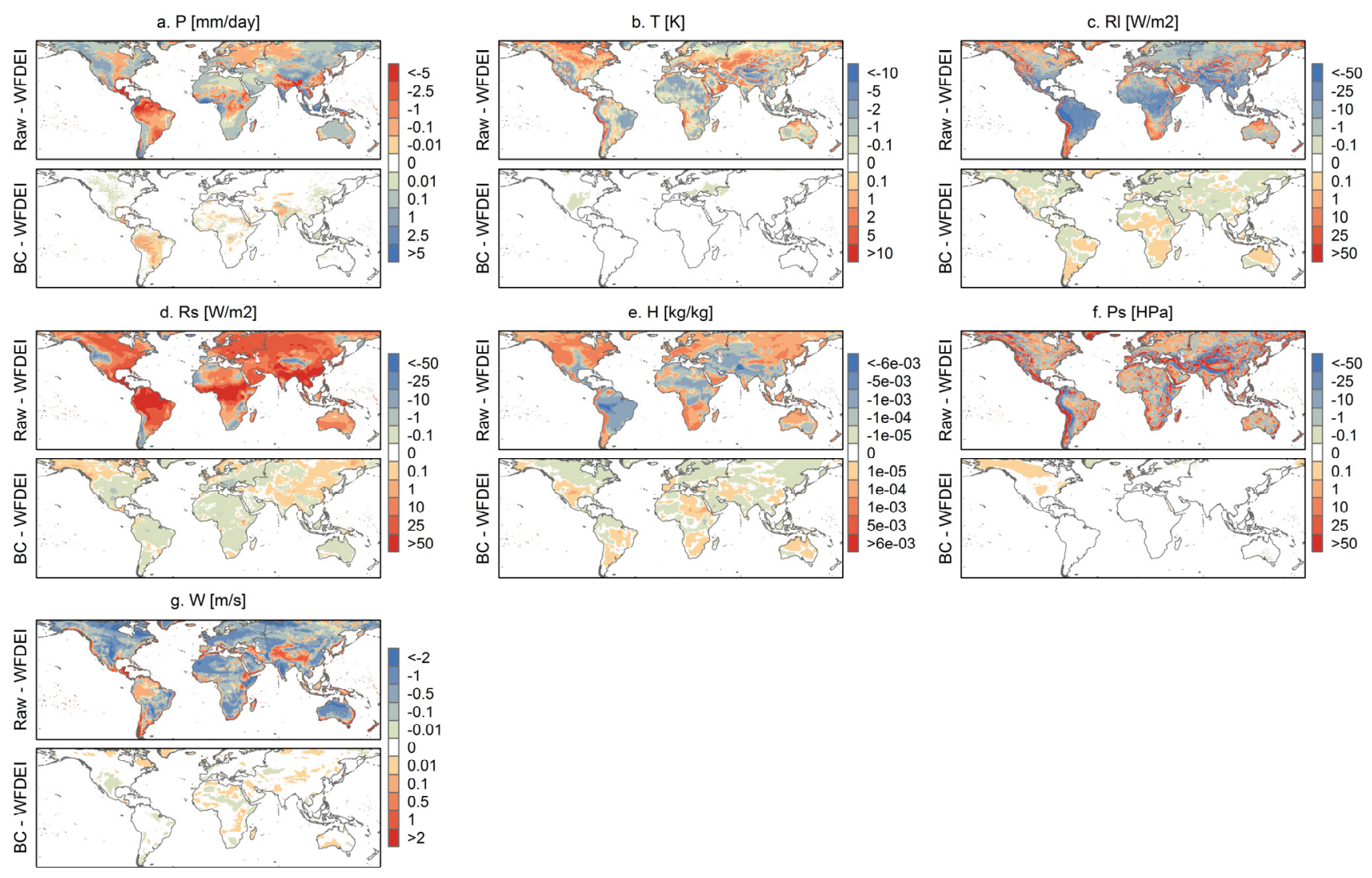

Figure S 2. Difference maps, showing initial (Raw-WFDEI) and remaining (BC-WFDEI) biases of the GCM ensemble forcing variables: a.Precipitation, b.Temperature, c.Longwave downward radiation, d.Shortwave downward radiation, e.Specific humidity, h.Surface pressure, g.Wind. Differences are calculated between the June-July-August averages (JJA) of the 1981-2010 period. 


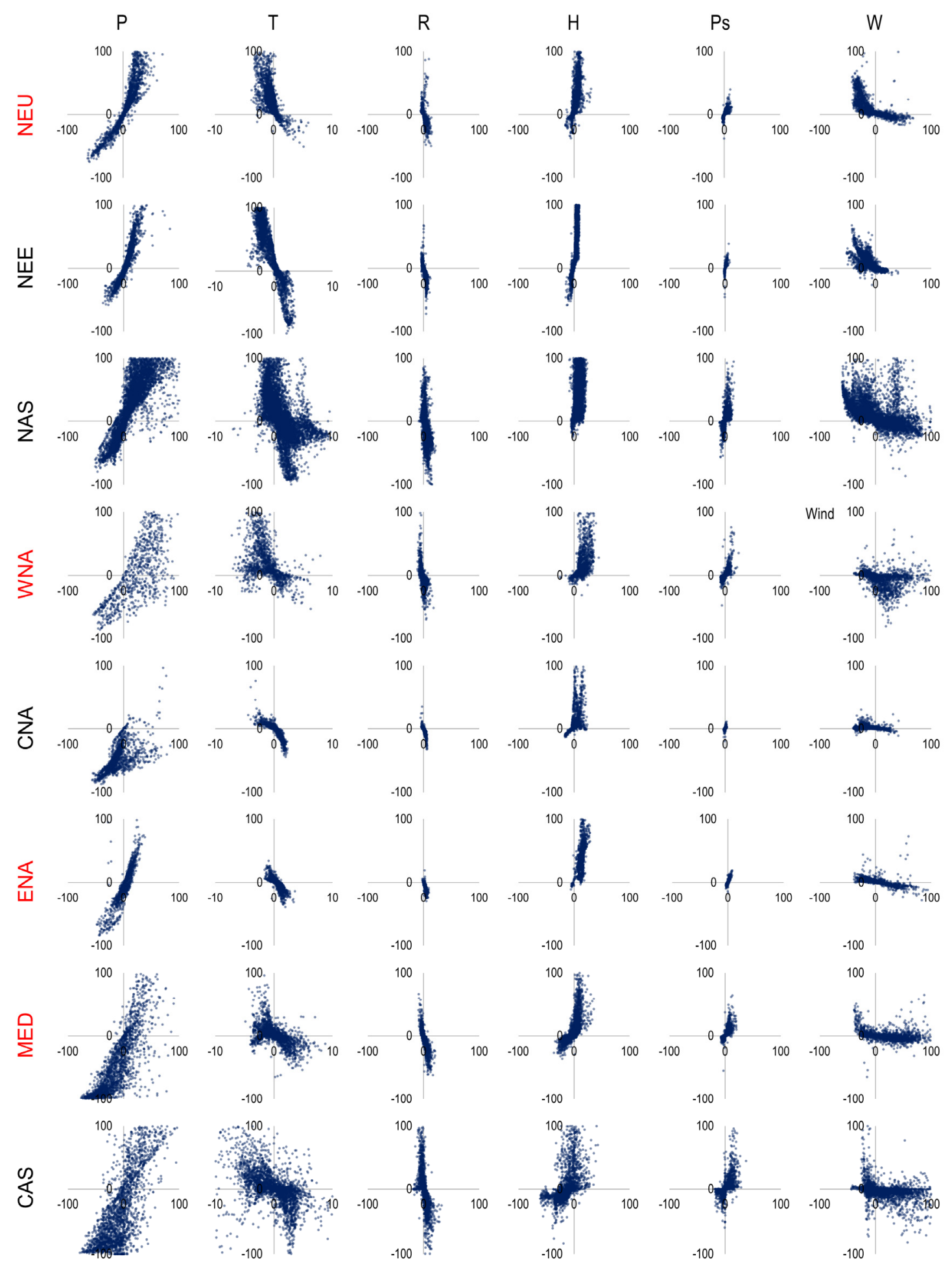

Figure $S 3$. Scatterplots of relative changes in forcing variable $(\Delta \mathrm{V}, \mathrm{x}$ axis) and corresponding relative changes in runoff ( $\triangle R F, y$ axis), for all the forcing variables and for the 24 regions. In each panel, each dot represents the $\Delta R F / \Delta V$ relationship of each land grid box in the examined region. The regions names in red colour correspond to the selected focus regions that are presented in the main paper. 

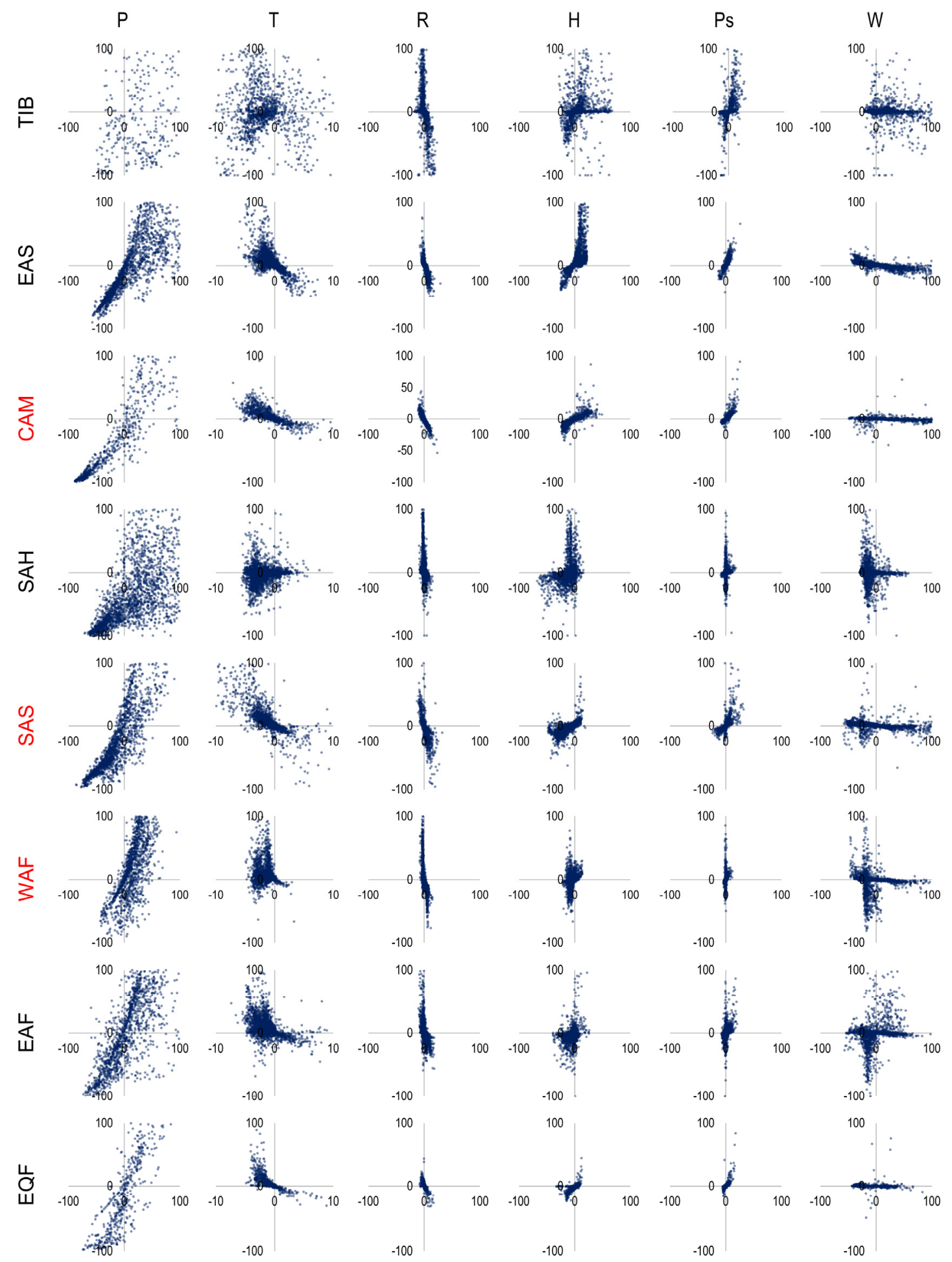

Figure S 3 (continued). 

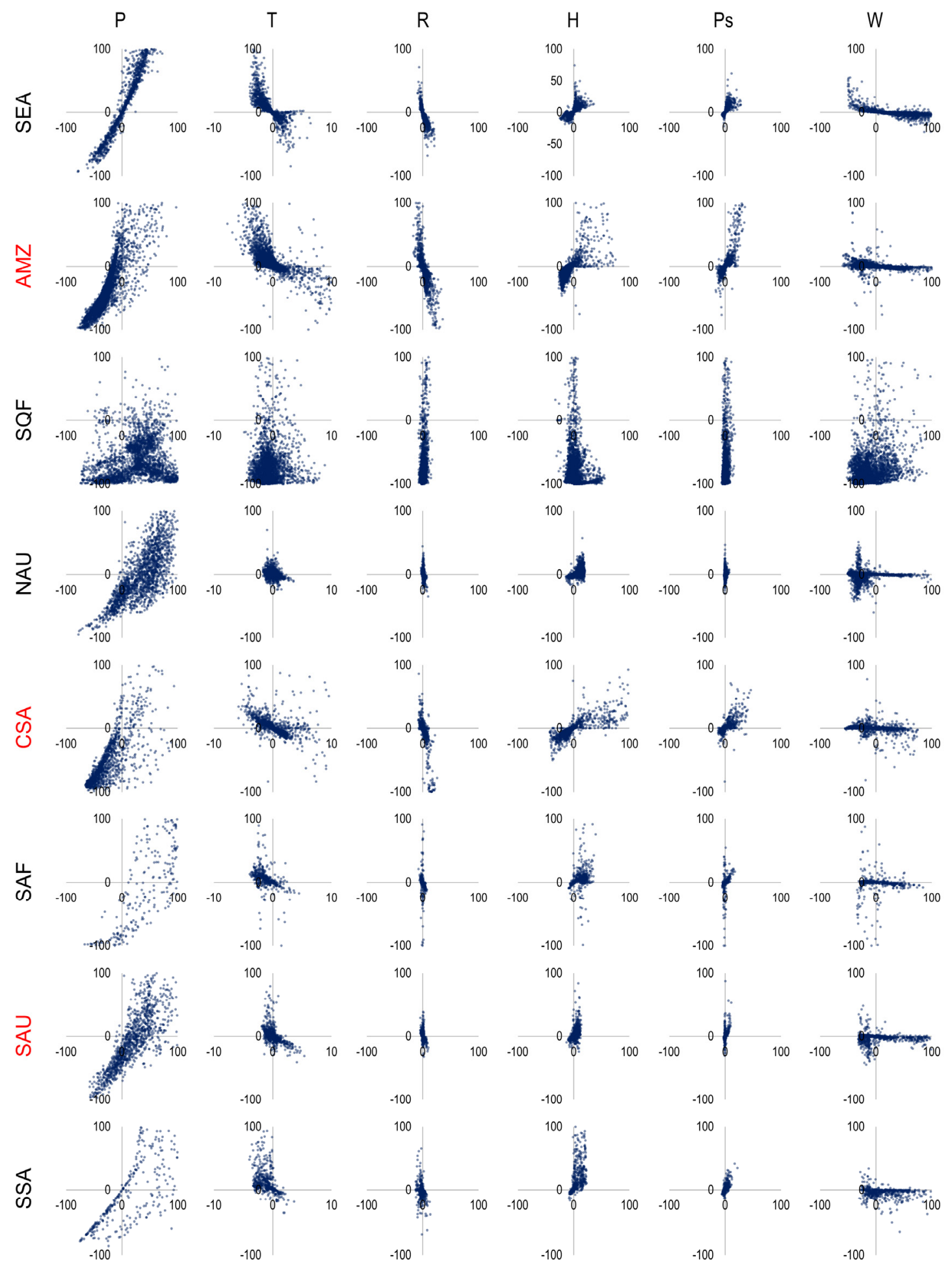

Figure S 3 (continued). 


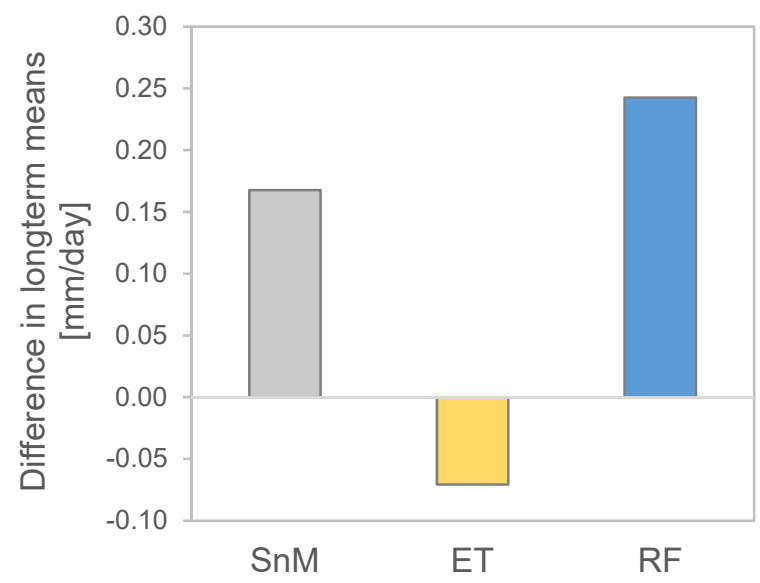

Figure S 4. Difference between the long term means (of the 1981-2010 period) of three fluxes (SnM:snowmelt, ET: evapotranspiration and RF:runoff), forced with raw and bias corrected humidity (forced with Raw H- forced with $\mathrm{BC} \mathrm{H})$. The fluxes are calculated for a representative grid box with center location at 60.25 Longitude and 60.25 Latitude.

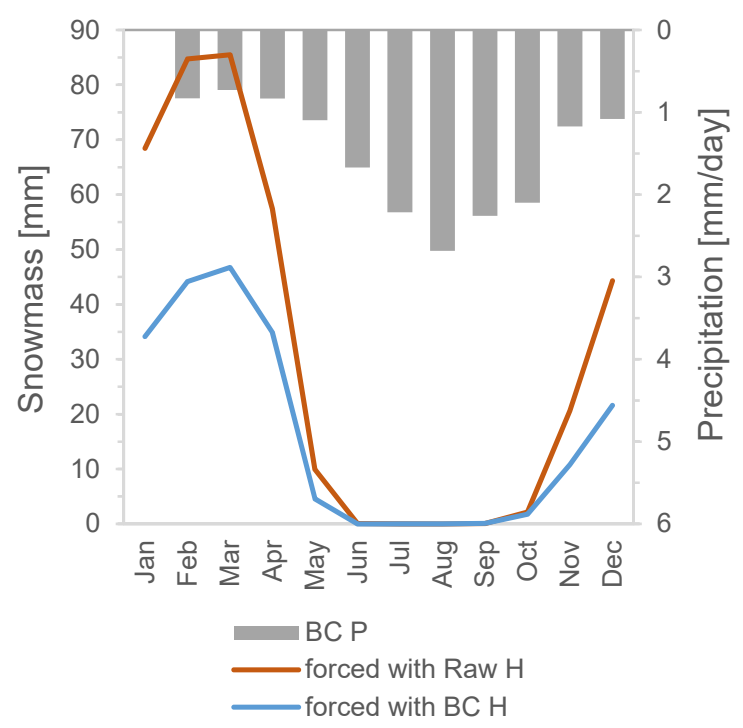

Figure S 5. Annual cycle of JULES' snowmass, forced with raw and bias corrected humidity [mm/day] and bias corrected precipitation (common forcing for both runs). Annual cycles are calculated from the 1981-2010 period, for a representative grid box with center location at 60.25 Longitude and 60.25 Latitude. 
a. WFDEI

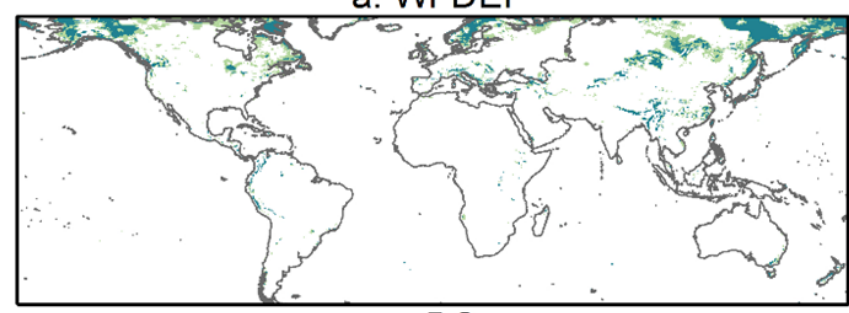

c. BC

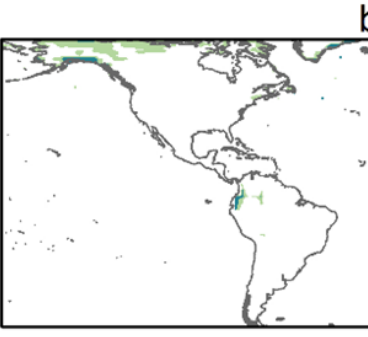

d. $\mathrm{NobcH}$

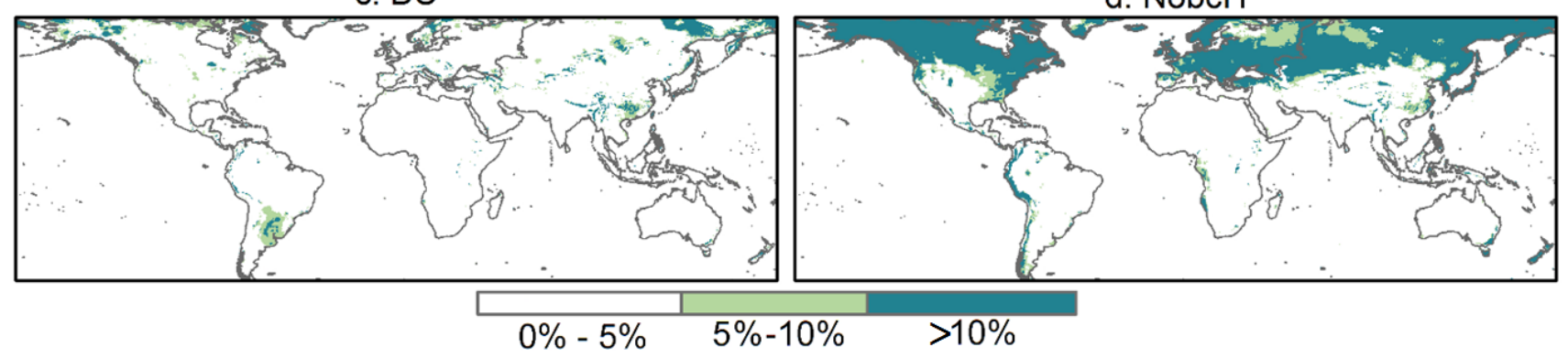

Figure S 6. Fraction of time under supersaturated air conditions (Relative humidity $>100 \%$ ), calculated from specific humidity $H$, temperature $T$ and surface pressure Ps for: a. WFDEI data, b. Raw GCM data, c. BC GCM

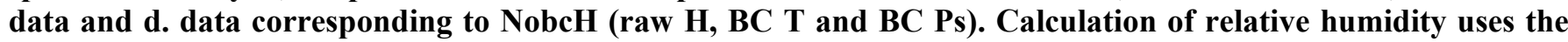
Clausius-Clapeyron equation. Fraction of time refers to the historical period 1981-2010. 